\title{
Ability in Using Chinese Adverb "Tongchang" and "Changchang" of STBA-PIA Second Year Student 2017/2018 Academic Year
}

\author{
Yenny Marlim \\ STBA-PIA, Medan, Sumatera Utara \\ marlimyenny@gmail.com \\ Yanti \\ STBA-PIA, Medan, Sumatera Utara \\ kimyanti96@gmail.com
}

\begin{abstract}
The research is aimed at discovering the ability of STBA-PIA Second Year Students 2017/2018 Academic Year in using the Chinese Adverb “Tongchang 通常” and “Changchang 常常”. This research uses a descriptive quantitive design method with a total population of 67 students. As the total population is under 100, the whole students are taken as the research sample. Students' ability in using both Chinese Adverbs “Tongchang 通常” and “Changchang 常常” is measured by using the test in which is the research instrument. The validity and reliability of the research instrument are examined by using content validity and internal reliability. Internal reliability is examined by using Kuder Richardson 20 formula. The reliability of the instrument shows the coefficient of 0.618 in which may be interpreted as quite a strong value. After passing the reliability score, the instrument is given a test to students. The result of the test is the ability of STBA-PIA second-year students in using the Chinese Adverb "Tongchang 通常" and "Changchang 常常" can be categorized as quite competent as $61.18 \%$ of students reach the average score of 74.72 for "Tongchang 通常" and $63.18 \%$ of students reach the average score of 72.04 for "Changchang 常常”.
\end{abstract}

Keywords: Cognitive Ability, Chinese Adverb, 通常 (tongchang), 常常 (changchang). 


\section{JOURNAL OF LANGUAGE, LITERATURE, AND TEACHING (JLLTE)}

Vol. 2 No. 3, December 2020 - March 2021, pp. 13-19

\section{前言}

李德律和金德厚 (2010) 认为副词是一般用在动词、形容词等等。毛悦与刘长正 （2014）认为副词限制、修饰动词或形容词性词语，表示程度、范围、时间等意义。

林建鸿（2016）对学生使用时间副词“通常”与“常常”的偏误进行了研究, 研究结果 显示学生的偏误情况为误加偏误（20\%）、误代偏误（45\%）、错序偏误（35\%），而 产生偏误的因素有语际迁移和语内迁移。汉语副词 “通常” 与 “常常” 的语义相近, 但在 语用方面有所不同。因此, 本研究目的是了解亚院二年级学生使用汉语副词 “通常” 与 “常常” 能力情况。

\section{副词概念}

毛悦和刘长正 (2014) 认为副词限制、修饰动词或形容词性词语, 表示程度、范围、 时间等意义。副词可以分为 9 类: 一、表示程度: “很、非常、十分” 等; 二、表示范围: “都、全、大概” 等; 三、表示时间: “刚、才、忽然” 等; 四、表示频率: “又、再、 也” 等; 五、表示处所：“处处、到处、随处” 等; 六、表示肯定：“一定、必须、必然” 等; 七、表示否定: “不、没、没有” 等; 八、表示方式: “一起、互相、特意” 等; 九、 表示语气: “难道、到底、也许” 等。汉语 “通常”与 “常常” 是时间副词。

\section{“通常”与 “常常” 的异同}

在《现代汉语词典》中，“常常”的释义是：（事情的发生）不止一次，而且时间 相隔不久。“通常” 的释义是：一般情况下行为事情有规律地发生。周小兵 (1994, 转引 自林建鸿，2016）解释 “通常” 和 “常常” 的异同可以从谓语前后、时间前后和副词的分 别来看。以下是两者的异同:

“通常” 和 “常常” 位于谓语之前

“常常” 可以跟单独的谓词结合, “通常”一般不行。 


\section{JOURNAL OF LANGUAGE, LITERATURE, AND TEACHING (JLLTE)}

Vol. 2 No. 3, December 2020 - March 2021, pp. 13-19

例：小李常常锻炼。

*小李通常锻炼。

常常” 可以跟动宾离合词或动宾词组结合, “通常”一般不行。

例: 小王常常洗澡。

*小王通常洗澡。

当 “常常” 和 “通常” 放在谓词之前, 表示方式、地点等意思的时候, 这两个词都可

以用。例：黄先生常常坐出租车回家。

黄先生通常坐出租车回家。

（2）“通常” 和 “常常” 位于时间前后

“常常” 和 “通常” 都可以放在时间词之前。

例: 他常常 9 点钟吃早饭。

他通常 9 点钟吃早饭。

当 “常常” 和 “通常” 放在时间之后, 这两个词的分布不一样。

例： a. 他星期天常常去公园散步。

b. 他星期天通常去公园散步。

c. 小敏去年常常坐公共汽车上班。

d. *小敏去年通常坐公共汽车上班。

例（a）和（b） “常常” 和 “通常” 都可以用, 而例（c）和（d）讲的是过去的情况, 一 般不可以用 “通常”。

“常常” 和 “通常” 都可以在时段时间词之前出现。

例: a. 他胃不好, 常常一天吃四顿。

b. 他胃不好, 通常一天吃四顿。

c. *他常常一年体检两次。

d. 他通常一年体检两次。 


\section{JOURNAL OF LANGUAGE, LITERATURE, AND TEACHING (JLLTE)}

Vol. 2 No. 3, December 2020 - March 2021, pp. 13-19

例（a）和（b） “常常” 和 “通常” 放在时段时间词的之前都可以用，而例（c）和（d） 是与长时段发生的情况相关，所以一般不可以用 “常常”，只能用 “通常”。

“常常” 和 “通常” 都可以在时段时间词之后出现。

例：他胃不好，一天常常吃四顿。

他胃不好，一天通常吃四顿。

（3）“通常” 和 “常常” 与搭配词 “不”、“都” 的用法

“常常” 和 “通常” 都可以在否定词 “不”之前出现。

例: 小成常常不在家吃午饭。

小成通常不在家吃午饭。

“常常” 可以在否定词 “不”、“没”、“没有”之后出现, 而 “通常” 却不可以在 “不”、“没”、“没有”之后出现。

例：我没有常常在饭馆吃饭。

*我没有通常在饭馆吃饭。

“通常” 可以用在表示总括的 “都” 之前, 而 “常常”一般不行。

例: *小李常常都骑摩托车去学校。

小李通常都骑摩托车去学校。

能力理论

Robbins (2007:57) 认为, 能力是对工作中能完成任何一项任务的人的评价。Waruwu (2010：47）认为能力可分为三部分：一、认知能力（Cognitive ability）, 包含思考 能力、幻想、志愿、远见、确定目标而定计划; 二、情感能力（Affective ability）, 包含于他人建立关系的能力; 三、精神能力 (Physchomotorability), 包含人类的行为 和行动。本研究所考察的能力情况属于认知能力。

Bloom (1956, 转引自许庆洲, 2017) 将认知能力可分成 6 个层次:

(1) 知识 (Knowledge) 


\section{JOURNAL OF LANGUAGE, LITERATURE, AND TEACHING (JLLTE)}

Vol. 2 No. 3, December 2020 - March 2021, pp. 13-19

包含辨别能力和记住名称、定义事实、建议、方针、排列、方法学、原则等等。举 例来说, 但被要求说明行政管理的素质时, 处于这个等级的人将能够良好分析出质产品最 低品质标准。

（2）理解 (Comprehension)

某个人对某件事的认知或理解能力是在他对那件事知道和记住之后。换句 来说, 理解的解释即是知道某件事和能从各方面个看到事实。一个参加受训的学员对事物 的理解是要他能够用自己的话解释和更进一步分析那件事的真相。

(3) 运用 (Application)

在这个等级内, 一个人具有能力说明他的倡议、程序、方针、公式、理论和其他在 学习过程需要具备的条件。

(4) 分析 (Analysis)

在分析的等级, 一个人将具有能力对递进的信息加以分析和分门别类, 并将信息加 以缩小, 以辩清它的方针和关系, 并能够认清和辨别以出复杂故事当然结构原因和结果。

(5) 综合 (Synthesis)

比分析更高一等级, 一个处于合成等级的人员将有能力说明一出故事的结构和方针, 实际上先前是无法看到的, 并具备能力认清资料或信息以便产生解决问题的门径。

(6) 评价 (Evaluation)

有能力对某项门径, 倡议, 方法学等利用一项合适或标准的途径以肯定效应或效益 的价值观。

\section{研究方法}

\section{总体与样本}

本文的研究设计类型是定量描述法。这项研究的总体是亚院二年级的学生，共有四 个班 67 人。Arikunto（2009：112）认为倘若研究总体少于 100 人, 研究样本就采取所有 的研究总体为样本。由于本研究的总体少于 100 人, 于是本研究的样本乃是 67 人。

\section{工具}




\section{JOURNAL OF LANGUAGE, LITERATURE, AND TEACHING (JLLTE)}

Vol. 2 No. 3, December 2020 - March 2021, pp. 13-19

本研究工具是测试。Arikunto（2013：193）测试是一系列的问题或练习题用于测量 个人或团体的技能、知识和能力。本研究使用的测试内容有 30 道题。

\section{数据分析}

本研究的工具效度和信度测验是使用内容效度和内在信度。测试内容来源于棉兰亚 洲国际友好学院《初级精读 I 》、《初级精读 II 》和《初级阅读 II 》的课程内容。内在 信度采用 KR20 公式, 信度系数为 0.618 (可靠性高)。数据分析技术采用描述性统计。

\section{研究结果}

\section{学生使用 “常常”、“通常” 的能力情况}

通过测试得知亚院二年级学生使用 “通常”、“常常” 的能力情况属于及格。学生使 用副词 “通常” 的平均分数是 74.72 和使用副词 “常常” 的平均分数是 72.04 。

\section{学生使用 “通常” 的能力情况}

学生使用副词 “通常” 的能力情况为 14 名学生分数介于 90 至 100 , 属于优秀 (20.89\%)； 17 名学生分数介于 80 至 89 , 属于良好（25.37\%）；10 名学生分数介于 70 至 79, 属于及格（14.92\%）；26 名学生分数 69 分以下，属于不及格（38.80\%）。从学生 得分情况可得知, 41 名学生（优秀、良好、及格）已经较好地掌握使用副词 “通常” 的 能力 (61. 18\%), 而其余的 26 名学生还没有掌握使用副词 “通常” 的能力。数据统计显示, 学生使用副词 “通常” 的平均数为 74.72 , 这意味着亚洲国际友好学院二年级学生使用副 词 “通常” 的能力属于及格。

\section{学生使用 “常常” 的能力情况}

学生使用副词 “常常” 的能力情况为 8 名学生分数介于 90 至 100 , 属于优秀 (11. 94\%)； 25 名学生分数介于 80 至 89 , 属于良好 (37. $31 \%) ； 10$ 名学生分数介于 70 至 79, 属于及格（14.92\%）；24 名学生分数 69 分以下，属于不及格（35.82\%）。从学生 


\section{JOURNAL OF LANGUAGE, LITERATURE, AND TEACHING (JLLTE)}

Vol. 2 No. 3, December 2020 - March 2021, pp. 13-19

得分情况可得知, 43 名学生（优秀、良好、及格）已经较好地掌握使用副词 “常常” 的 能力 (64.18\%) ，而其余的 24 名学生还没有掌握使用副词 “常常” 的能力。数据统计显 示, 学生使用副词 “通常” 的平均数为 72.04 , 这意味着亚洲国际友好学院二年级学生使 用副词 “常常” 的能力属于及格。

\section{研究讨论}

林建鸿（2016）的研究结果显示汉语副词 “通常”与 “常常” 的所出现错误类型有 三种：误加偏误（20\%）、误代偏误（45\%）、错序偏误（35\%），其中的误代偏误是最 为常见。而本研究显示 $38.80 \%$ 和 $35.82 \%$ 的亚洲国际友好学院二年级学生还没掌握好使用 副词 “通常” 和 “常常” 的能力。由此可见, 本研究结果支持了林建鸿的研究结果。

\section{参考文献}

书籍

[1] 黄伯荣. 现代汉语（上册） [M]. 北京: 北京大学出版社, 2012 .

[2] 黄伯荣和廖序东. 现代汉语（上册） [M]. 北京：高等教育出版社， 2010.

[3] 李德律和金德厚. 汉语语法教学 [M]. 北京: 北京语言大学出版社, 2010.

[4] 毛悦和刘长正. 汉语作为第二语言教学: 汉语要素教学 [M]. 北京: 外语教学与研究出 版社, 2014 .

[5] 周小兵. 对外汉语教学导论 [M]. 北京: 商务印书馆出版社, 2009.

Arikunto, S. 2009. Prosedur Penelitian Suatu Pendekatan Praktik. Jakarta: PT. Rineka Cipta. Arikunto, S. 2013. Prosedur Penelitian Suatu Pendekatan Praktik.Jakarta: PT. Rineka Cipta. Robbins. 2007. Meningkatkan Kemampuan Menulis Karangan Narasi. Jakarta: PT. Indeks. Waruwu, F. 2010. Membangun Budaya Berbasis Nilai. Yogyakarta: Kanisius. 


\section{JOURNAL OF LANGUAGE, LITERATURE, AND TEACHING (JLLTE)}

Vol. 2 No. 3, December 2020 - March 2021, pp. 13-19

\section{论文或期刊}

[1] 林建鸿. 亚院二年级学生使用时间副词 “通常” 与 “常常” 的偏误分析 [D]. 棉兰: 亚 洲国际友好学院，2016.

[2] 许庆洲. 学校初中一年级学生使用标点符号能力研究分析 [D]. 棉兰: 亚洲国际友好学 院, 2017.

[3] 杨智渤. “通常”与 “常常” 的凸显差异 $[\mathrm{J}]$. 汉语学习, 2013.

[4] 庄婷婷. 论 “通常”与 “常常”的异同 [J]. 剑南文学经典教苑, 2012. 